\title{
Identification of the Bacterial Gene in Patients Who Repeatedly Develop Streptococcal Pharyngitis ${ }^{*}$
}

\author{
Yuichiro Tsuji , Ichihashi Izumi
}

Department of Pediatrics, Sempos Tokyo Takanawa Hospital, Tokyo, Japan.

Email: "yuitsuji@ybb.ne.jp

Received September $20^{\text {th }}, 2012$; revised October $20^{\text {th }}$, 2012; accepted November $25^{\text {th }}, 2012$

\begin{abstract}
Background: We aimed at determining whether the pathogenic bacteria at the onset of disease are genetically different and whether this affects future choice of the therapeutic methods against group A $\beta$-hemolytic streptococcal acute pharyngitis/tonsillitis. Methods: A pharynx swab was collected from pediatric patients who visited our hospital. The swab was cultured, and hemolytic streptococcus was detected 230 times. We isolated pathogenic bacteria of patients infected more than once and examined the bacteria using pulse-field gel electrophoresis. Results: Based on gene search results, we found that if the period of developing relapse was within 1 month from the first infection, all patients had the same gene. However, all patients in whom reinfection occurred after 6 months or later had different pertinent genes. Conclusions: The number of relapse/reinfection is significant for this disease, and considerably caution is essential for its treatment. No changes to antibacterial drug administration may be necessary for the second administration unless more than 6 months have passed since the first infection.
\end{abstract}

Keywords: Bacterial Gene; Cefcapene Pivoxil; PFGE (Pulse-Field Gel Electrophoresis); Reinfection; Streptococcal Pharyngitis

\section{Introduction}

The number of pediatric patients who repeatedly develop acute pharyngitis/tonsillitis is large when this disease is caused by group A $\beta$-hemolytic streptococcus (hemolytic streptococcus). Information on whether the pathogenic bacteria at onset are genetically different is useful when choosing therapeutic methods and for determining whether the therapy was effective in eradicating the bacteria. Thus, we isolated pathogenic bacteria from the pharynx of pediatric patients who developed pharyngitis/ tonsillitis more than once and examined whether the pathogenic bacteria were identical using pulse-field gel electrophoresis (PFGE).

\section{Methods}

Subjects were ambulant pediatric patients who visited the pediatrics department of our hospital from February 2007 to March 2009 and were suspected of having acute streptococcal pharyngitis/tonsillitis.

Pharynx swabs were collected from the patients and cultured, and hemolytic streptococcus was detected 230 times in total. At the time of diagnosis, a rapid diagnostic

\footnotetext{
*Disclosure: the authors report no conflicts of interest in this work.

\#Corresponding author.
}

kit was simultaneously used. In case in which the rapid diagnosis was positive, we diagnosed the condition as hemolytic streptococcus infection before obtaining the results of pharynx culture, and antibacterial drugs were administered. Referring to the Diagnosis Guideline of Pediatric Respiratory Organ Infectious Disease in 2007 [1] and taking the compliance of pediatric patients into consideration, the patients were administered cefcapene pivoxil 3 times per day (t.i.d.) at a dose of $3 \mathrm{mg} /(\mathrm{kg} \cdot$ dose) for five days.

\subsection{PFGE Procedure}

After cultured at $35^{\circ} \mathrm{C}$ for $20 \mathrm{hr}$ in blood agar medium, bacteria were suspended in saline-EDTA $(0.15 \mathrm{M} \mathrm{NaCl}$, $10 \mathrm{mM}$ EDTA), collected, and washed. Pett IV (1 M $\mathrm{NaCl}, 10 \mathrm{mM}$ EDTA, pH 8.0) solution was added to the pellet, and the bacteria were re-suspended. After an equal volume of $2 \%$ low-melting point agarose solution was added to the bacterial suspension, the suspension was dispensed in plug molds and solidified. After the plug was taken out and placed in microtubes, a lysis solution was added to the tubes. Bacteriolysis was conducted by incubating the tubes at $35^{\circ} \mathrm{C}$ overnight.

After the lysis solution was removed, ESP $(0.25 \mathrm{M}$ EDTA $\mathrm{pH}$ 8.0, $1 \% \mathrm{~N}$-lauroyl sarcosine sodium, 1.5 
$\mathrm{mg} / \mathrm{ml}$ proteinase $\mathrm{K}$ ) was added, and the protein was degraded by incubating the tubes at $50^{\circ} \mathrm{C}$ overnight.

The plug was placed in TE (10 mM Tris- $\mathrm{HCl} \mathrm{pH} 8.0,1$ $\mathrm{mM}$ EDTA $\mathrm{pH}$ 8.0), to which phenylmethyl sulfonyl fluoride (PMSF) was added at a final concentration of 1 $\mathrm{mM}$ and incubated at ambient temperature for $3 \mathrm{hr}$. The plug was then washed with TE. Using Sma I or Sfi I, restriction enzyme treatment was performed at $30^{\circ} \mathrm{C}$ for 8 hr. Subsequently, electrophoresis was conducted using $0.8 \%$ agarose with the CHEF DR-III system (BIO-Rad, Hercules, CA, USA).

After stainig with GelStar, the gel was observed on a transilluminator, a photograph was taken, and band patterns were compared. Negative control was Y: Yeast chromosomes, Saccharomyces cerevisiae.

Measurements were performed in Mitsubishi Chemical Medience Corporation (Tokyo, Japan).

\subsection{Subject Cases}

Eighty-five male subjects and 97 female subjects (total. 182 ) were examined. The average patient age was $6.59 \pm$ 2.81 ( $\pm 1 \mathrm{SD})$.

The number of culture-positive cases was 230. Sixteen male children and 15 female children (total. 31) were found to be culture-positive more than once. Since a smear electrophoretic pattern was observed on PFGE in one of these cases, we were unable to examine this case, and therefore, the total number of cases examined was 30 .

\section{Results}

Table 1 shows the number of cases.

Cases of infections with more than one occurrence accounted for approximately $17 \%$ of cases during the investigation period.

Case presentation:

Figure 1 shows a representative case referred to as Case 1 . In this case, pharynx culture-positive results were observed 4 times within 5 months, and PFGE showed the

Table 1. Number of infections in subject cases.

\begin{tabular}{cc}
\hline Times & Cases \\
\hline 6 & 1 \\
5 & 1 \\
4 & 2 \\
3 & 6 \\
2 & 21 \\
1 & 151 \\
total & 182 \\
\hline
\end{tabular}

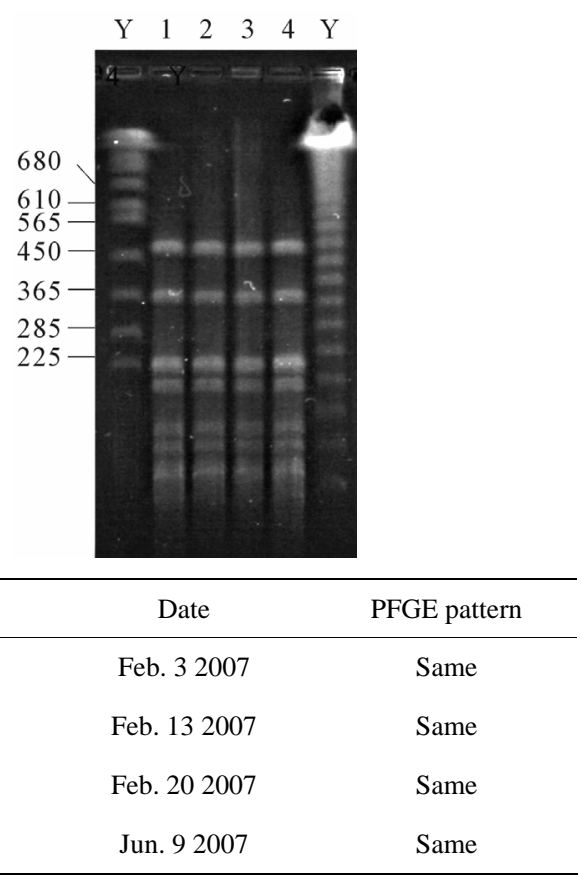

Figure 1. Case 1 is 5 years old male whose PFGE of pathogenic bacteria from his pharynx shows all same pattern. PFGE = pulse-field gel electrophoresis. $\mathrm{Y}=$ yeast chromosomes, Saccharomyces cerevisiae.

same pattern.

Figure 2 (Case 2) shows a positive pharynx culture that was confirmed 4 times within 26 months. Although the same patterns were observed in PFGE within 1 month, the PFGE patterns were different after an interval of 24 months.

Figure 3 (Case 3) shows that pharynx culture-positive was seen 5 times within approximately 1 year. Although the same patterns were seen on PFGE within 2 weeks, all other PFGE results showed different patterns, indicating that the patient results showed different patterns, indicating that the patient was infected with 4 types of hemolytic streptococcus strains.

Investigation of gene patterns in patients with infection occurring more than once (shown in Table 2).

Within 1 month, all cases showed the same genetic patterns. However, after an interval of 6 months, all cases showed different genetic patterns. Between 1 and 6 months, the same gene pattern as well as different gene patterns were found. This difference was statistically significant $(p<0.001)$. We selected the statistical significance level was $\mathrm{p}<0.05$. The official approval used t-test. All analyses were conducted using a commercially available software program (Stat View, version 5.0; Abacus Concepts Inc, California, USA).

\section{Discussion}

Since the same genetic pattern was observed in all culture- 


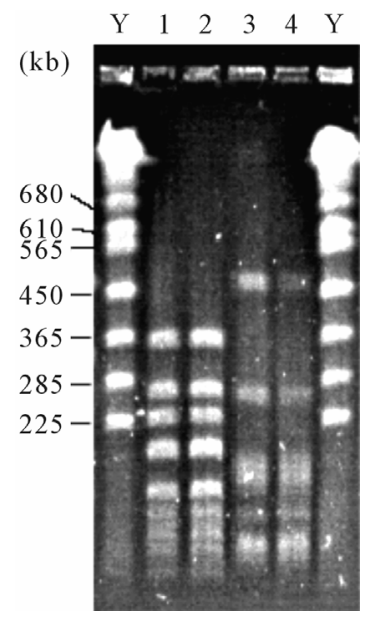

\begin{tabular}{ccc}
\hline Lane & Date & PFGE pattern \\
\hline 1 & Feb. 4 2009 & A \\
2 & Jan. 6 2009 & A \\
3 & Jan. 15 2007 & B \\
4 & Des. 27 2006 & B \\
\hline
\end{tabular}

Figure 2. Case 2 is 5 years old female whose PFGE of pathogenic bacteria from her pharynx shows two pairs of patterns. PFGE = pulse-field gel electrophoresis. $Y=$ yeast chromosomes, Saccharomyces cerevisiae.

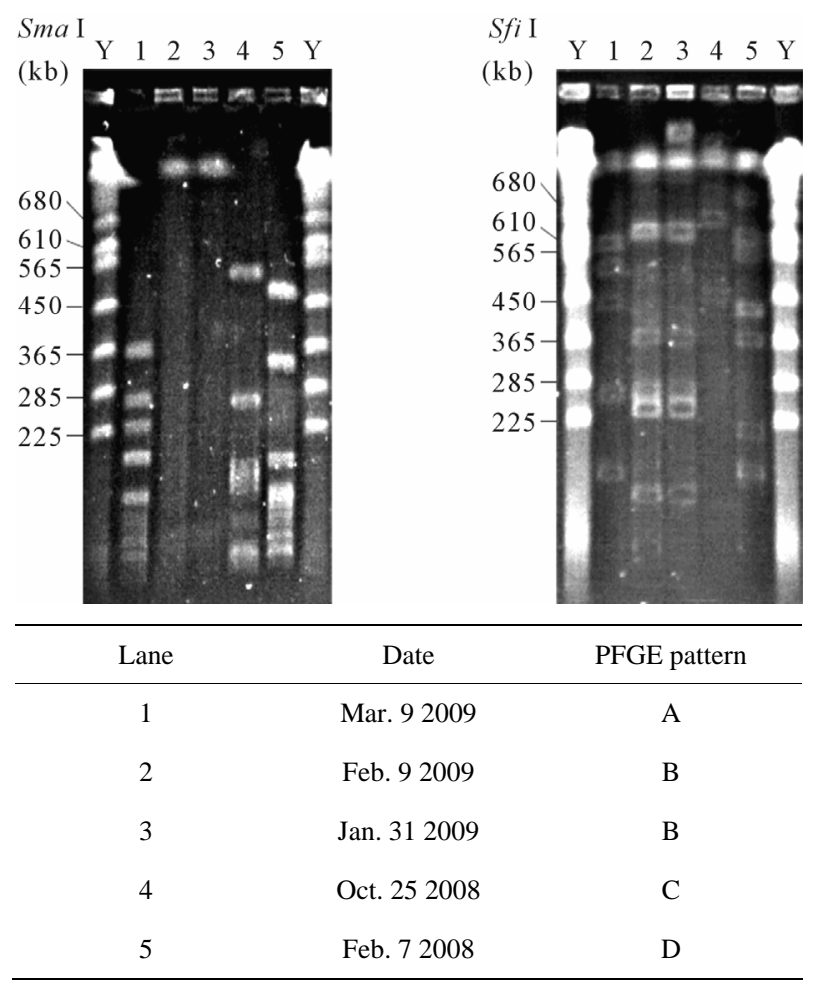

Figure 3. Case 3 is 8 years old female whose PFGE of pathogenic bacteria from her pharynx shows four different patterns. PFGE = pulse-field gel electrophoresis. $\mathbf{Y}=$ yeast chromosomes, Saccharomyces cerevisiae.
Table 2. Investigation on gene patterns in patients suffering from the infection more than once.

\begin{tabular}{ccc}
\hline Interval & $\begin{array}{c}\text { No. of patients } \\
\text { same pattern }\end{array}$ & $\begin{array}{c}\text { No. of patients } \\
\text { different patterns }\end{array}$ \\
\hline$\sim 2$ weeks & 12 & 0 \\
3 weeks - 4 weeks & 8 & 0 \\
1 month - 3 months & 1 & 3 \\
4 months - 6 months & 1 & 3 \\
7 months & 0 & 11 \\
\hline
\end{tabular}

positive cases with reinfection twice or more times within 1 month, therapeuric methods must be altered in such cases, based on "prescription example to the cases of bacteriological relapse" described in the Diagnosis Guideline of Pediatric Respiratory Organ Infectious Disease in 2007 [1]. In contrast, since all cases showed different gene patterns regarding infection that developed after interval of 6 months, the patients were likely to be infected with different hemolytic streptococcal strains; hence, there is no need to change antibacterial drugs administered. In case in which patients experienced infection twice or more times, 1 month or later but within 6 months of the first infection, treatment decision must be made by comprehensively taking into account factors of the host side, epidemic situation, and infection history of pediatric patients. In this study, the proportion of plural pharynx culture-positive cases was as high as approximately 17\% (Table 2). It has been thought that this result is attributable to the fact that our hospital is a community-based one in which the rate of return to clinic is predominantly high. Patients will not likely visit emergency hospitals or university hospitals at the relapse/ reinfection, but visit primary care doctors near their homes. Because our hospital is a community-based general hospital, the rate of return to clinic is predominantly high, which is likely to contribute the present results. At the first diagnosis itself, we inform and help parents understand that relapse/reinfection can occur and that a re-diagnosis is required if symptoms appear again. The extent to which parents understood our explanation may have affected the number of plural pharynx culture-positive cases.

Additionally, the long period of investigation, over 2 years, is likely to affect the present results. Patients are likely to experience the second or subsequent development of streptococcal pharyngitis/tonsillitis for a longer period of time at a markedly high probability.

With respect to the choice of therapeutic agents, there are reports that a therapeutic method using cefem drugs is superior to penicillin drugs in bacterial eradication [2-4], and higher efficacy of cefcapene pivoxil has been 
reported by Sakata et al., who examined the antibacterial effect on hemolytic streptococcus [5]. Sunakawa et al. reported that the bacterial eradication of hemolytic streptococcus was $100 \%$ in their study on the efficacy, safety, and pharmacokinetics of cefcapene pivoxil granules for children/infants in pediatric patients with infectious disease [6]. In addition, Pichichero et al. reported that the number of reinfections was significantly larger in a group treated with penicillin antibacterial drugs [7]. Considering these reports, the large number of the second or subsequent infections in our hospital is not significantly attributable to the antibacterial strength of the selected drugs. Table 2 shows that the ratio of relapse within 1 month relative to the rate of multiple infections is $91 \%$ (20/22). Considering that relapse observed within 1 month was caused by the same bacteria, the relapse rate during this period was estimated to be approximately $11 \%$. Therefore, the rate of clinically ineffective cases is thought to be approximately coincident to this value. This value is not largely different from that described in another report [8]. In the investigation in our hospital in which 166 cases were administered cefdinir $3 \mathrm{mg} /(\mathrm{kg} /$ dose) t.i.d. (9 mg/[kg/day]), the relapse rate was $18.1 \%$; thus, similar results were obtained as that of a previous study [9].

Martin et al. reported that carriers were most often school-aged children with group A streptococci infection and frequently switched emm types [10]. These results may be related to our results. Although it was different from infected patients and carriers, the finding of switching after a constant period with gene constitution of hemolytic streptococcus is consisitent with that of our report.

Furthermore, by molecular typing using restriction fragment length polymorphism analysis of total DNA, Fitoussi et al. reported that bacteriological failures were due to homologous strains in $70 \%$ of cases and the acquisition of new strains in 30\% of cases [11]. Although their study was different from ours in terms of the treatment regimen and period of sampling, it was comparable to our report in terms of relapse/reinfection after antibiotic therapy where the same strain accounts for most of the infections.

Studies on plural relapse/reinfection within a short term that was caused by the same strain have been already conducted in other regions, but the general trend in Japan was demonstrated in this study more definitely. Our report will be useful for determining appropriate treatments for reinfection in Japan.

We used the PFGE technique for the analysis of the gene pattern of the hemolytic streptococcus. Musser et al. described that PFGE was useful for the analysis of the gene pattern of the hemolytic streptococcus [12].

In the present study, the so-called "shuttle infection" with the same strain carried by a family member could not be ruled out. Additional studies are needed to address this point.

This study was performed after obtaining the approval from the ethics committee established in our hospital. Additionally, in the bacterial gene search, consent by the family was obtained in writing before beginning the search.

\section{Acknowledgements}

This work was supported by the clinical research fund in Sempos Tokyo Takanawa Hospital.

\section{Summary}

Based on the our gene search in streptococcal pharyngitis patients, all cases had the same gene within 1 month, on the other hand all cases had the different gene after 6 months or later from the first infection.

\section{REFERENCES}

[1] Japan Pediatric Respiratory Tract Disease Association/ Japan Pediatric infectious Disease Association, “Diagnosis Guideline of Pediatric Respiratory Organ Infectious Disease,” Kyowa Kikaku, Tokyo, 2007.

[2] M. E. Pichichero and P. A. Margolis, "A Comparison of Cephalosporins and Penicillins in the Treatment of Group A Beta-Hemolytic Streptococcal Pharyngitis: A MetaAnalysis Supporting the Concept of Microbial Copathogenicity," Pediatric Infectious Disease Journal, Vol. 10, No. 4, 1991, pp. 275-281. doi:10.1097/00006454-199104000-00002

[3] J. R. Casey and M. E. Pichichero, "Meta-Analysis of Cephalosporin versus Penicillin Treatment of Group A Streptococcal Tonsillopharyngitis in Children,” Pediatrics, Vol. 113, No. 4, 2004, pp. 866-882. doi:10.1542/peds.113.4.866

[4] S. Tsuchida and T. Tomofumi, "Cefteram Pivoxil FiveDay Administration therapy on Group A $\beta$-Hemolytic Streptococcus infectious Disease,” Journal of Pediatric, Vol. 64, 2001, pp. 1605-1608.

[5] H. Sakata, "Comparative Study of 5-Day Cefcapene-Pivoxil and 10-Day Amoxicillin or Cefcapene-Pivoxil for Treatment of Group A Streptocpccal Pharyngitis in Children," Journal of Infection and Chemotherapy, Vol. 14, No. 3, 2008, pp. 208-212. doi:10.1007/s10156-008-0597-0

[6] S. Keisuke, T. Nishimura, M. Takashi, I. Naoichi, Y. Takayoshi, W. Ehiro and F. Yoshitomo, "Investigation on Efficacy, Safety, and Pharmacokinetics of Granules of Cefcapene Pivoxil for Child in Pediatric Patients with Infectious Disease," Journal of Chemical Engineering of Japan, Vol. 54, 2006, pp. 465-477.

[7] M. E. Pichichero, J. L. Green, A. B. Francis, S. M. Marsocci, M. L. Murphy, W. Hoeger, C. Noriega, A. Sorrento and J. Gootnick, "Recurrent Group A Streptococcal Tonsillopharyngitis," Pediatric Infectious Disease Journal, 
Vol. 17, No. 9, 1998, pp. 809-815. doi:10.1097/00006454-199809000-00012

[8] K. Toshimi, "A Clinical Study on Cases of Group A Hemolytic Streptococcus Infectious Disease Whose Bacterial Eradication Failed," Journal of Pediatric, Vol. 20, 2000, pp. 117-120.

[9] T. Yuichiro, I. Izumi, M. Koji and I. Kazuo, "Efficacy of Cephalosporin Antibiotics in Acute Streptococcal Pharyngitis/Tonsillitis Patients," Journal of the Japan Pediatric Society, Vol. 113, 2009, p. 318.

[10] J. M. Martin, M. Green, K. A. Barbadora and E. R. Wald, "Group A Streptococci among School-Aged Children: Clinical Characteristics and the Carrier State,” Pediatrics, Vol. 114, No. 5, 2004, pp. 1212-1219. doi:10.1542/peds.2004-0133

[11] F. Fitoussi, R. Cohen, G. Brami, C. Doit, N. Brahimi, F. Rocque and E. Bingen, "Molecular DNA Analysis for Differentiation of Persistence or Relapse from Recurrence in Treatment Failure of Streptococcus Pyogenes Pharyngitis," European Journal of Clinical Microbiology and Infectious Diseases, Vol. 16, No. 3, 1997, pp. 233-237. doi:10.1007/BF01709587

[12] J. M. Musser, V. Kapur and J. Szeto, "Genetic Diversity and Relationships among Streptococcus Pyogenes Strains Expressing Serotype M1 Protein: Recent intercontinental Spread of Subclone Causing Episodes of Invasive Disease," Infection and Immunity, Vol. 32, No. 3, 1995, pp. 994-1003. 